\title{
Existence and Numerical Solution of the Volterra Fractional Integral Equations of the Second Kind
}

\author{
Abdon Atangana ${ }^{1}$ and Necdet Bildik ${ }^{2}$ \\ ${ }^{1}$ Institute for Groundwater Studies, Faculty of Natural and Agricultural Sciences, University of the Free State, \\ Bloemfontein 9300, South Africa \\ ${ }^{2}$ Department of Mathematics, Faculty of Art \& Sciences, Celal Bayar University, Muradiye Campus, 45047 Manisa, Turkey
}

Correspondence should be addressed to Abdon Atangana; abdonatangana@yahoo.fr

Received 26 July 2013; Accepted 18 September 2013

Academic Editor: Hossein Jafari

Copyright (C) 2013 A. Atangana and N. Bildik. This is an open access article distributed under the Creative Commons Attribution License, which permits unrestricted use, distribution, and reproduction in any medium, provided the original work is properly cited.

This work presents the possible generalization of the Volterra integral equation second kind to the concept of fractional integral. Using the Picard method, we present the existence and the uniqueness of the solution of the generalized integral equation. The numerical solution is obtained via the Simpson 3/8 rule method. The convergence of this scheme is presented together with numerical results.

\section{Introduction}

The integral equations form an important part of applied mathematics, with links with many theoretical fields, especially with practical fields [1-3]. The Volterra integral [13] equations were introduced by Vito Volterra and then studied by Traian Lalescu in his 1908 thesis. Volterra integral equations find application in demography, the study of viscoelastic materials, and in insurance mathematics through the renewal equation. Fredholm equations [4] arise naturally in the theory of signal processing, most notably as the famous spectral concentration problem popularized by David Slepian [4]. They also commonly arise in linear forward modeling and inverse problems. Throughout the last decade, physicists and mathematicians have paid attention to the concept of fractional calculus [5-9]. Actually, real problems in scientific fields such as groundwater problems, physics, mechanics, chemistry, and biology are described by partial differential equations or integral equations. Many scholars have shown with great success the applications of fractional calculus to groundwater pollution and groundwater flow problems [59], acoustic wave problems [10], and others [11-14]. There are also several iteration methods for solving fractional integral equations like homotopy decomposition method [15-17], variational iteration method [18-20], Adomian decomposition method [21, 22], and others [23, 24]. But in this work, we will make use of the numerical method called the Simpson $3 / 8$ rule. The general equation under analysis here is given as

$$
\begin{array}{r}
F(t)=G(t)+\frac{1}{\Gamma(\alpha)} \int_{a}^{t}(t-\tau)^{\alpha-1}[K(t, \tau) F(\tau)] d \tau, \\
0<\tau \leq t \leq T ; \quad \alpha \geq 0 .
\end{array}
$$

Here,

$$
\begin{aligned}
& F(t)=\left[f_{1}(t), f_{2}(t), f_{3}(t), f_{4}(t), \ldots f_{n}(t)\right]^{T}, \\
& G(t)=\left[g_{1}(t), g_{2}(t), g_{3}(t), g_{4}(t), \ldots g_{n}(t)\right]^{T}, \\
& K(t, \tau, F(\tau)) \\
& =\left(\begin{array}{c}
K_{1}\left(\left[t, \tau, f_{1}(\tau), f_{2}(\tau), f_{3}(\tau), f_{4}(\tau), \ldots f_{n}(\tau)\right]\right) \\
K_{2}\left(\left[t, \tau, f_{1}(\tau), f_{2}(\tau), f_{3}(\tau), f_{4}(\tau), \ldots f_{n}(\tau)\right]\right) \\
\vdots \\
K_{n}\left(\left[t, \tau, f_{1}(\tau), f_{2}(\tau), f_{3}(\tau), f_{4}(\tau), \ldots f_{n}(\tau)\right]\right)
\end{array}\right) .
\end{aligned}
$$

For the rest of this paper, we assume that $a<\tau \leq t \leq T<\infty$. In this paper, system (1) can be linear or nonlinear. 


\section{Basic Information about the Fractional Calculus}

Definition 1. A real function $f(x), x>0$, is said to be in the space $C_{-\mu}, \mu \in \mathbb{R}$, if there exists a real number $p>\mu$, such that $f(x)=x^{p} h(x)$, where $h(x) \in C[0, \infty)$, and it is said to be in space $C_{\mu}^{m}$ if $f^{(m)} \in C_{\mu}, m \in \mathbb{N}$.

Definition 2. The Riemann-Liouville fractional integral operator of order $\alpha \geq 0$, of a function $f \in C_{-} \mu, \mu \geq-1$, is defined as

$$
\begin{gathered}
J^{\alpha} f(x)=\frac{1}{\Gamma(\alpha)} \int_{0}^{x}(x-t)^{\alpha-1} f(t) d t, \quad \alpha>0, \quad x>0, \\
J^{0} f(x)=f(x) .
\end{gathered}
$$

Properties of the operator can be found in [25-29]; we mention only the following.

$$
\begin{gathered}
\text { For } f \in C_{\mu}, \mu \geq-1, \alpha, \beta \geq 0 \text { and } \gamma>-1, \\
J^{\alpha} J^{\beta} f(x)=J^{\alpha+\beta} f(x), \quad J^{\alpha} J^{\beta} f(x)=J^{\beta} J^{\alpha} f(x), \\
J^{\alpha} x^{\gamma}=\frac{\Gamma(\gamma+1)}{\Gamma(\alpha+\gamma+1)} x^{\alpha+\gamma} .
\end{gathered}
$$

The fractional derivative of $f(x)$ in the Caputo sense is defined as

$$
\begin{array}{r}
{ }_{a}^{c} D_{x}^{\alpha} f(x)=J^{m-\alpha} D^{m} f(x) \\
=\frac{1}{\Gamma(m-\alpha)} \int_{a}^{x}(x-t)^{m-\alpha-1} f^{m}(t) d t \\
m-1<\alpha \leq m, m \in \mathbb{N}, \\
x>0, f \in C_{-1}^{\mu} .
\end{array}
$$

Also, we need here two of its basic properties.

Lemma 3. If $m-1<\alpha \leq m, m \in \mathbb{N}$, and $f \in C_{\mu}^{m}, \mu \geq-1$, then

$$
\begin{gathered}
{ }_{a}^{c} D_{x}^{\alpha} J^{\alpha} f(x)=f(x), \\
J^{\alpha} D_{0}^{\alpha} f(x)=f(x)-\sum_{k=0}^{m-1} f^{(k)}\left(0^{+}\right) \frac{x^{k}}{k !}, \quad x>0 .
\end{gathered}
$$

\section{Existence and Uniqueness Analysis}

The analysis of the existence and the uniqueness analysis are important aspects that must be investigated before the presentation of the solution. One of the most common techniques used to achieve this is the fixed point theorem technique. To prove the existence and uniqueness of the solution of the system (1), we make use of the method of successive approximation, also called the Picard method [30]. This consists of simple iterations. Before we start this proof, we will assume the following.
First, making use of the vector norm, we assume that

$$
\begin{gathered}
\|F(t)\|=\max _{1 \leq i \leq n}\left|f_{i}(t)\right|, \\
\|K(s, t)\|=\max _{1 \leq i \leq n} \sum_{k}^{n}\left|k_{i, j}(s, t)\right| .
\end{gathered}
$$

In this method, we assume that the following iteration can be used to provide a series solution of the problem under investigation:

$$
F_{n}(t)=G(t)+\frac{1}{\Gamma(\alpha)} \int_{a}^{t}(t-\tau)^{\alpha-1}\left[K(t, \tau) F_{n-1}(\tau)\right] d \tau .
$$

It also assumes that the initial component of the series solution is given as

$$
F_{0}(t)=G(t) .
$$

Let us in addition put the difference between the consecutive components as

$$
\delta_{n}(t)=F_{n}(t)-F_{n-1}(t)
$$

It is very easy to see that

$$
F_{n}(t)=\sum_{i=0}^{n} \delta_{i}(t)
$$

\subsection{Existence and Uniqueness of the Linear Volterra Fractional Integral Equations of the Second Kind}

Theorem 4 (see [31]). Under the conditions that the vector functions $G(t)$ and $K(s, t)$ are continuous $0 \leq a<\tau \leq t \leq T<$ $\infty$, then, the system of Volterra fractional integral equations of the second kind (1) has a unique continuous solution for $0 \leq$ $a<t \leq T<\infty$.

The proof is similar to the one in [31].

Theorem 5. Assuming that the system (1) has a unique solution, say $F(t)$ in $a<t \leq T$, such that $K(t, \tau) F(\tau)$ is absolutely fractionally integrable, and if in addition

$$
\|G(t)\|<g(t), \quad\|K(t, s)\|<k(t, s),
$$

providing that $k$ and $g$ are continuous functions, then it is possible to find a function, say $f(t)$, such that

$$
\|F(t)\|<f(t)
$$

where $f(t)$ is the continuous function solutions of

$$
f(t)=g(t)+\frac{1}{\Gamma(\alpha)} \int_{a}^{t}(t-\tau)^{\alpha-1}[K(t, \tau) f(\tau)] d \tau .
$$

Proof. From (1), applying the vector norm on both sides, we obtain the following:

$$
\|F(t)\|=\left\|G(t)+\frac{1}{\Gamma(\alpha)} \int_{a}^{t}(t-\tau)^{1-\alpha}[K(t, \tau) F(\tau)] d \tau\right\| .
$$


Now, making use of the inequality triangular, we obtain the following:

$$
\begin{gathered}
\|F(t)\| \leq\|G(t)\|+\left\|\frac{1}{\Gamma(\alpha)} \int_{a}^{t}(t-\tau)^{\alpha-1}[K(t, \tau) F(\tau)] d \tau\right\|, \\
\|F(t)\| \leq\|G(t)\|+\frac{1}{\Gamma(\alpha)} \int_{a}^{t}(t-\tau)^{\alpha-1}[\|K(t, \tau)\|\|F(\tau)\|] d \tau .
\end{gathered}
$$

Thus, making use of the hypothesis, we obtain

$$
\|F(t)\|<g(t)+\frac{1}{\Gamma(\alpha)} \int_{a}^{t}(t-\tau)^{\alpha-1}[k(t, \tau)\|F(\tau)\|] d \tau .
$$

If now the difference between (14) and (17) gives

$$
\begin{aligned}
f(t)-\|F(t)\|> & \frac{1}{\Gamma(\alpha)} \\
& \quad \times \int_{a}^{t}(t-\tau)^{\alpha-1} \\
& \quad \times[k(t, \tau)(f(t)-\|F(\tau)\|)] d \tau,
\end{aligned}
$$

since $f(a)-\|F(a)\|>0$ and also $k(t, \tau)$ is a continuous positive function, it is then true to conclude that

$$
f(t)-\|F(t)\|>0, \quad \text { for } t \leq T,
$$

which concludes the proof.

Theorem 6. Under the condition that $G(t), K(t, s), \Delta G(t)$, and $\Delta K(t, s)$ are smooth functions and bounded one has

$$
\begin{aligned}
& \|K(t, \tau)\| \leq k, \quad\|\Delta K(t, \tau)\| \leq \Delta k, \\
& \|G(t)\| \leq g, \quad\|\Delta G(t)\| \leq \Delta g .
\end{aligned}
$$

Let $F_{\text {exact }}(t)$ be the exact solution of

$$
\begin{aligned}
& F_{\text {exact }}(t)= G(t)+\Delta G(t) \\
&+\frac{1}{\Gamma(\alpha)} \int_{a}^{t}(t-\tau)^{\alpha-1} d \tau \\
& \quad \times\left[(K(t, \tau)-\Delta K(t, \tau)) F_{\text {exact }}(\tau)\right] ;
\end{aligned}
$$

then,

$$
\begin{aligned}
& \left\|F_{\text {exact }}(t)-F(t)\right\| \\
& \quad \leq\left\{\Delta g+\Delta k t(g+\Delta g) E_{\alpha}((k+\Delta k) t)\right\} \times E_{\alpha}\left(k^{-\alpha} t\right) \\
& \quad=O(\Delta g)+O(\Delta k),
\end{aligned}
$$

with $F_{\text {exact }}$ is the solution of system (1).

Proof. Since $G(t), K(t, s)$ are smooth functions and bounded, using Theorem 4 , there exists a positive smooth function $f(t)$ such that

$$
f(t)=g(t)+\frac{1}{\Gamma(\alpha)} \int_{a}^{t}(t-\tau)^{\alpha-1}[k f(\tau)] d \tau .
$$

For simplicity, we chose $a=0$; then,

$$
f(t)=g(t)+\frac{k}{\Gamma(\alpha)} \int_{0}^{t}(t-\tau)^{\alpha-1}[f(\tau)] d \tau .
$$

Using the methodology of the homotopy decomposition method, we arrive at the following exact solution:

$$
f(t)=g E_{\alpha}\left(k^{-\alpha} t\right),
$$

where

$$
E_{\alpha}\left(k^{-\alpha} t\right)=\sum_{n=0}^{\infty} \frac{k^{n} t^{n \alpha}}{\Gamma[1+n \alpha]}
$$

known as the Mittag-Leffler function. Therefore,

$$
\|F(t)\|<g E_{\alpha}\left(k^{-\alpha} t\right) .
$$

With the above in hand, it is very easy to show that

$$
\left\|F_{\text {exact }}(t)\right\| \leq(g+\Delta g) E_{\alpha}\left((k+\Delta k)^{-\alpha} t\right) .
$$

Since $F_{\text {exact }}(t)$ is the approximate solution of system (1), then it follows that

$$
F_{\text {exact }}(t) \approx G(t)+\frac{1}{\Gamma(\alpha)} \int_{0}^{t}(t-\tau)^{\alpha-1}\left[K(t, \tau) F_{\text {exact }}(\tau)\right] d \tau \text {. }
$$

So the error in the approximation can be represented as

$$
\begin{aligned}
R(t)=G(t)+\frac{1}{\Gamma(\alpha)} \int_{0}^{t}( & t-\tau)^{\alpha-1} \\
\times & {\left[K(t, \tau) F_{\text {exact }}(\tau)\right] d \tau } \\
& -F_{\text {exact }}(t) .
\end{aligned}
$$

Now replacing $F_{\text {exact }}(t)$ as in (21), we obtain

$$
R(t)=-\Delta G(t)+\frac{1}{\Gamma(\alpha)} \int_{0}^{t}(t-\tau)^{\alpha-1}\left[\Delta K(t, \tau) F_{\text {exact }}(\tau)\right] d \tau
$$

Then, the difference between the exact solution and the approximate solution can be obtained as

$$
\begin{aligned}
F(t)-F_{\text {exact }}( & () \\
=R(t)+\int_{0}^{T} & \frac{1}{\Gamma(\alpha)} \\
& \times(t-\tau)^{\alpha-1}[\Delta K(t, \tau) \\
& \left.\times\left[F(t)-F_{\text {exact }}(\tau)\right]\right] d \tau
\end{aligned}
$$

so that

$$
\begin{aligned}
& \left\|F(t)-F_{\text {exact }}(t)\right\| \\
& <\Delta g+\Delta k t \max (\|F(t)\|) \\
& \leq\left\{\Delta g+\Delta k t(g+\Delta g) \times E_{\alpha}((k+\Delta k) t)\right\} E_{\alpha}\left(k^{-\alpha} t\right) \\
& =O(\Delta g)+O(\Delta k),
\end{aligned}
$$

which completes the proof. 
3.2. Existence and Uniqueness of the Nonlinear Volterra Fractional Integral Equations of the Second Kind. In this case, the nonlinear Volterra fractional integral equations of the second kind considered here are

$$
\begin{gathered}
F(t)=G(t)+\frac{1}{\Gamma(\alpha)} \int_{a}^{t}(t-\tau)^{\alpha-1}[K(t, \tau, F(\tau))] d \tau, \\
0<\tau \leq t \leq T ; \alpha \geq 0 .
\end{gathered}
$$

$F(t), G(t)$, and $K(t, \tau, F(\tau))$ have the same form as in the previous subsection; also the norm used in the previous section is maintained.

In analogy with what was done in Section 3.1, we define the iteration formula as

$$
F_{n}(t)=G(t)+\frac{1}{\Gamma(\alpha)} \int_{a}^{t}(t-\tau)^{\alpha-1}[K(t, \tau, F(\tau))] d \tau,
$$

with initial component

$$
F_{0}(t)=G(t)
$$

Similarly, the difference between the consecutive terms is given as

$$
\begin{aligned}
F_{n}(t)-F_{n-1}(t) & \\
=\frac{1}{\Gamma(\alpha)} \int_{a}^{t}(t-\tau)^{\alpha-1}[ & K\left(t, \tau, F_{n-1}(\tau)\right) \\
& \left.-K\left(t, \tau, F_{n-2}(\tau)\right)\right] d \tau .
\end{aligned}
$$

We will perhaps recall that in case the kernel $K$ satisfies the Lipchitz conditions, we have the following inequality:

$$
\left\|K\left(t, \tau, F_{1}\right)-K\left(t, \tau, F_{2}\right)\right\| \leq H\left\|F_{1}-F_{2}\right\|,
$$

with of course $H$ being a real positive number not depending on the parameters $t, \tau, F_{1}$, and $F_{2}$.

As in Section 3.1, we put

$$
\delta_{n}(t)=F_{n}(t)-F_{n-1}(t) .
$$

Again, we have that

$$
F_{n}(t)=\sum_{i=0}^{n} \delta_{i}(t)
$$

Then,

$$
\begin{gathered}
\left\|\delta_{n}(t)\right\| \\
=\| \frac{1}{\Gamma(\alpha)} \int_{a}^{t}(t-\tau)^{\alpha-1}\left[K\left(t, \tau, F_{n-1}(\tau)\right)\right. \\
\left\|\delta_{n}(t)\right\| \leq \frac{1}{\Gamma(\alpha)} \int_{0}^{t}(t-\tau)^{\alpha-1} \\
\left.\times \| K\left(t, \tau, F_{n-2}(\tau)\right)\right] d \tau \|
\end{gathered}
$$

In case the Lipchitz condition is satisfied by the kernel, we have the following inequality:

$$
\begin{aligned}
& \left\|\delta_{n}(t)\right\| \leq \frac{1}{\Gamma(\alpha)} \int_{a}^{t}(t-\tau)^{\alpha-1} \\
& \times \| K\left(t, \tau, F_{n-1}(\tau)\right) d \tau, \\
& -K\left(t, \tau, F_{n-2}(\tau)\right) \| \\
& \left\|\delta_{n}(t)\right\| \leq \frac{1}{\Gamma(\alpha)} \int_{a}^{t}(t-\tau)^{\alpha-1}\left\|\delta_{n-1}\right\| d \tau .
\end{aligned}
$$

We will then present the following theorem.

Theorem 7. Under the conditions that $G(t), K(t, \tau, F)$ are continuous in $0<\tau \leq t \leq T<\infty,-\infty<F<\infty$ and the kernel satisfies the Lipchitz condition, that is, $\| K\left(t, \tau, F_{1}\right)-$ $K\left(t, \tau, F_{2}\right)\|\leq H\| F_{1}-F_{2} \|$, then, (34) has a unique solution.

Proof. From (43), it follows that

$$
\left\|\delta_{n}(t)\right\| \leq \max _{0<t \leq T}\|G(t)\| \frac{\left(H^{-\alpha} t\right)^{n \alpha}}{\Gamma(1+n \alpha)} ;
$$

therefore,

$$
F(t)=\sum_{i=0}^{n} \delta_{n}(t)
$$

exists and is a continuous function. However, to prove that the above function is the solution of the system of the nonlinear Volterra fractional integral equations (34) of the second kind, we let

$$
F(t)=F_{n}(t)-P_{n}(t)
$$

Now, using (36), we have the following equation:

$$
\begin{aligned}
& F(t)-F_{n}(t) \\
& =G(t)+\frac{1}{\Gamma(\alpha)} \int_{0}^{t}(t-\tau)^{\alpha-1} K\left(t, \tau, F(\tau)-P_{n}(\tau)\right) d \tau .
\end{aligned}
$$

It follows that

$$
\begin{gathered}
F(t)-G(t)-\frac{1}{\Gamma(\alpha)} \int_{0}^{t}(t-\tau)^{\alpha-1} K(t, \tau, F(\tau)) d \tau \\
=P_{n}(t)+\frac{1}{\Gamma(\alpha)} \int_{0}^{t}(t-\tau)^{\alpha-1} \\
\times K\left(t, \tau, F(\tau)-P_{n}(\tau)\right. \\
-K(t, \tau, F(\tau)) d \tau) .
\end{gathered}
$$

Now, applying the norm and Lipchitz condition, we arrive at the following inequality:

$$
\begin{aligned}
& \left\|F(t)-G(t)-\frac{1}{\Gamma(\alpha)} \int_{0}^{t}(t-\tau)^{\alpha-1} K(t, \tau, F(\tau)) d \tau\right\| \\
& \quad \leq\left\|P_{n}(t)\right\|+H t\left\|P_{n-1}(t)\right\| .
\end{aligned}
$$


Applying the limit on both sides of the above inequality when $n$ tends to infinity, the right-hand side tends to zero; then, $F(t)$ in (45) satisfies

$$
F(t)=G(t)+\frac{1}{\Gamma(\alpha)} \int_{0}^{t}(t-\tau)^{\alpha-1} K(t, \tau, F(\tau)) d \tau,
$$

and indeed it is the solution of (34).

We will now present the uniqueness of this solution. To achieve this, we assume that (34) has another solution, say $F_{1}(t)$; then,

$$
\begin{aligned}
& F(t)-F_{1}(t) \\
& \begin{aligned}
=\frac{1}{\Gamma(\alpha)} \int_{0}^{t}(t-\tau)^{\alpha-1} \\
\quad \times\left[K(t, \tau, F(\tau))-K\left(t, \tau, F_{1}(\tau)\right)\right] d \tau .
\end{aligned}
\end{aligned}
$$

Applying the norm and making use of the Lipchitz condition of the kernel, we arrive at

$$
\left\|F(t)-F_{1}(t)\right\| \leq H \int_{0}^{t}(t-\tau)^{\alpha-1}\left\|F(\tau)-F_{1}(\tau)\right\| d \tau,
$$

but $\left\|F(t)-F_{1}(t)\right\| \leq D$; then,

$$
\left\|F(t)-F_{1}(t)\right\| \leq D \frac{\left(H^{-\alpha} t\right)^{n \alpha}}{\Gamma(1+n \alpha)},
$$

for any $n$; then,

$$
F(t)=F_{1}(t)
$$

\section{Numerical Method to Solve the Volterra Fractional Integral Equations}

We consider the general form of the Volterra fractional integral equation as

$$
\begin{aligned}
F(t)=G(t)+\frac{1}{\Gamma(\alpha)} \int_{a}^{t}(t-\tau)^{\alpha-1} & \\
& \times[K(t, \tau, F(\tau))] d \tau, \\
0 & <\tau \leq t \leq T ; \quad \alpha \geq 0,
\end{aligned}
$$

where $G(t)$ is a known function, $F(t)$ is an unknown function to be determined, and the kernel is $K(t, y, f)$.

In numerical analysis, Simpson's rule is a method for numerical integration, the numerical approximation of definite integrals [32]. Simpson's rule also corresponds to the 3 -point Newton-Cotes quadrature rule. The method is credited to the mathematician Thomas Simpson (1710-1761) of Leicestershire, England. Simpson's rule is a staple of scientific data analysis and engineering. It is widely used, for example, by naval architects, to numerically integrate hull offsets and cross-sectional areas to determine volumes and centroids of ships or lifeboats [33].
4.1. Application of the Simpson 3/8 to Volterra Fractional Integral Equation. To use Simpson's rule here, we let $0=a<$ $t_{1}<t_{2}<t_{3} \cdots<t_{n}$ be a possible division of $[0, b]$, with step size $x_{i}=i k$ for $i=0,1, \ldots N$. We construct a block by block method that is the system of Volterra fractional integral equation (1) for $q>1$ simultaneous equations is then a set of $q$ simultaneous value of the function $F$. Without loss of generality, we consider $q=6$.

For the rest of the paper,

$$
(t-\tau)^{\alpha-1} K[t, \tau F(\tau)]=K_{\alpha}(t, \tau, F(\tau))
$$

will be called the fractional kernel. Having the fractional kernel in hand, system (1) can be rewritten as follows:

$$
F(t)=G(t)+\frac{1}{\Gamma(\alpha)} \int_{0}^{t}\left[K_{\alpha}(t, \tau, F(\tau))\right] d \tau .
$$

Now, if we set $t=t_{3 n+1}$ in the previous equation, we obtain

$$
\begin{aligned}
F_{1,3 n+1}(t)= & g_{1}\left(t_{3 n+1}\right) \\
& +\frac{1}{\Gamma(\alpha)} \int_{0}^{t_{3 n+1}} K_{\alpha(1,1)}\left[t_{3 n+1}, \tau, f_{1}(\tau)\right] d \tau \\
& +\frac{1}{\Gamma(\alpha)} \int_{0}^{t_{3 n}} K_{\alpha(1,2)}\left[t_{3 n+1}, \tau, f_{2}(\tau)\right] d \tau \\
& +\frac{1}{\Gamma(\alpha)} \int_{t_{3 n}}^{t_{3 n+1}} K_{\alpha(1,1)}\left[t_{3 n+1}, \tau, f_{1}(\tau)\right] d \tau \\
& +\frac{1}{\Gamma(\alpha)} \int_{t_{3 n}}^{t_{3 n+1}} K_{\alpha(1,2)}\left[t_{3 n+1}, \tau, f_{2}(\tau)\right] d \tau \\
F_{2,3 n+1}(t)= & g_{2}\left(t_{3 n+1}\right) \\
& +\frac{1}{\Gamma(\alpha)} \int_{0}^{t_{3 n+1}} K_{\alpha(2,1)}\left[t_{3 n+1}, \tau, f_{2}(\tau)\right] d \tau \\
& +\frac{1}{\Gamma(\alpha)} \int_{0}^{t_{3 n}} K_{\alpha(2,2)}\left[t_{3 n+1}, \tau, f_{2}(\tau)\right] d \tau \\
& +\frac{1}{\Gamma(\alpha)} \int_{t_{3 n}}^{t_{3 n+1}} K_{\alpha(2,1)}\left[t_{3 n+1}, \tau, f_{2}(\tau)\right] d \tau \\
& +\frac{1}{\Gamma(\alpha)} \int_{t_{3 n}}^{t_{3 n+1}} K_{\alpha(2,2)}\left[t_{3 n+1}, \tau, f_{2}(\tau)\right] d \tau
\end{aligned}
$$

From here if one integrates over the interval $\left[0, t_{3 n}\right]$, we can apply Simpson's $3 / 8$ rule, and also by integrating over $\left[t_{3 n}, t_{3 n+1}\right]$, one can calculate it by using a cubic interpolation. Then, we can have the following:

$$
\begin{gathered}
F_{1,3 n+1}\left(t_{3 n+1}\right) \\
=g_{1}\left(t_{3 n+1}\right)
\end{gathered}
$$




$$
\begin{aligned}
& +\frac{3 h}{8 \Gamma(\alpha)}\left(K_{\alpha(1,1)}\left(t_{3 n+1}, t_{0}, F_{1,0}\right)\right. \\
& +3 K_{\alpha(1,1)}\left(t_{3 n+1}, t_{1}, F_{1,1}\right) \\
& +3 K_{\alpha(1,1)}\left(t_{3 n+1}, t_{2}, F_{1,2}\right) \\
& +2 K_{\alpha(1,1)}\left(t_{3 n+1}, t_{3}, F_{1,3}\right)+\cdots \\
& \left.+K_{\alpha(1,1)}\left(t_{3 n+1}, t_{3 n}, F_{1,3 n}\right)\right) \\
& +\frac{3 h}{8 \Gamma(\alpha)}\left(K_{\alpha(2,1)}\left(t_{3 n+1}, t_{0}, F_{2,0}\right)\right. \\
& +3 K_{\alpha(2,1)}\left(t_{3 n+1}, t_{1}, F_{2,1}\right) \\
& +3 K_{\alpha(2,1)}\left(t_{3 n+1}, t_{2}, F_{2,2}\right) \\
& +2 K_{\alpha(2,1)}\left(t_{3 n+1}, t_{3}, F_{2,3}\right)+\cdots \\
& \left.+K_{\alpha(2,1)}\left(t_{3 n+1}, t_{3 n}, F_{2,3 n}\right)\right) \\
& +\frac{h}{8 \Gamma(\alpha)}\left(K_{\alpha(1,1)}\left(t_{3 n+1}, t_{3 n}, F_{1,3 n}\right)+3 K_{\alpha(1,1)}\right. \\
& \times\left(t_{3 n+1}, t_{3 n+1 / 3}, \frac{40}{81} F_{1,3 n}\right. \\
& \left.-\frac{20}{27} F_{1,3 n+1}-\frac{8}{27} F_{1,3 n+1}+\frac{5}{81} F_{1,3 n+3}\right) \\
& +3 K_{\alpha(1,1)} \\
& \times\left(t_{3 n+1}, t_{3 n+2 / 3}, \frac{14}{81} F_{1,3 n+1}\right. \\
& \left.-\frac{7}{27} F_{1,3 n+2}+\frac{4}{81} F_{1,3 n+3}\right) \\
& \left.+K_{\alpha(1,1)}\left(t_{3 n+1}, t_{3 n+1}, F_{1,3 n}\right)\right) \\
& +\frac{h}{8 \Gamma(\alpha)}\left(K_{\alpha(1,2)}\left(t_{3 n+1}, t_{3 n}, F_{2,3 n}\right)\right. \\
& +3 K_{\alpha(1,2)}\left(t_{3 n+1}, t_{3 n+1 / 3}, \frac{40}{81} F_{2,3 n}\right. \\
& F_{1,3 n+2}(t)=g_{1}\left(t_{3 n+2}\right) \\
& +\frac{1}{\Gamma(\alpha)} \int_{0}^{t_{3 n+2}} K_{\alpha(1,1)} \\
& \times\left[t_{3 n+2}, \tau, f_{1}(\tau)\right] d \tau \\
& +\frac{1}{\Gamma(\alpha)} \int_{0}^{t_{3 n}} K_{\alpha(1,2)} \\
& \times\left[t_{3 n+2}, \tau, f_{2}(\tau)\right] d \tau \\
& +\frac{1}{\Gamma(\alpha)} \int_{t_{3 n}}^{t_{3 n+2}} K_{\alpha(1,1)} \\
& \times\left[t_{3 n+1}, \tau, f_{1}(\tau)\right] d \tau \\
& +\frac{1}{\Gamma(\alpha)} \int_{t_{3 n}}^{t_{3 n+2}} K_{\alpha(1,2)} \\
& \times\left[t_{3 n+2}, \tau, f_{2}(\tau)\right] d \tau, \\
& F_{2,3 n+2}(t)=g_{2}\left(t_{3 n+1}\right) \\
& +\frac{1}{\Gamma(\alpha)} \int_{0}^{t_{3 n+2}} K_{\alpha(2,1)} \\
& \times\left[t_{3 n+2}, \tau, f_{2}(\tau)\right] d \tau \\
& +\frac{1}{\Gamma(\alpha)} \int_{0}^{t_{3 n}} K_{\alpha(2,2)} \\
& \times\left[t_{3 n+2}, \tau, f_{2}(\tau)\right] d \tau \\
& +\frac{1}{\Gamma(\alpha)} \int_{t_{3 n}}^{t_{3 n+2}} K_{\alpha(2,1)} \\
& \times\left[t_{3 n+2}, \tau, f_{2}(\tau)\right] d \tau \\
& +\frac{1}{\Gamma(\alpha)} \int_{t_{3 n}}^{t_{3 n+2}} K_{\alpha(2,2)} \\
& \times\left[t_{3 n+2}, \tau, f_{2}(\tau)\right] d \tau .
\end{aligned}
$$$$
-\frac{20}{27} F_{2,3 n+1}-\frac{8}{27} F_{2,3 n+2}
$$$$
\left.+\frac{5}{81} F_{2,3 n+3}\right)
$$$$
+3 K_{\alpha(1,2)}\left(t_{3 n+1}, t_{3 n+2 / 3}, \frac{14}{81} F_{2,3 n+1}\right.
$$$$
\left.-\frac{7}{27} F_{2,3 n+2}+\frac{4}{81} F_{2,3 n+3}\right)
$$$$
\left.+K_{\alpha(1,2)}\left(t_{3 n+1}, t_{3 n+1}, F_{2,3 n}\right)\right) \text {. }
$$

From here if one integrates over the interval $\left[0, t_{3 n}\right]$, we can apply Simpson's $3 / 8$ rule, and also by integrating over $\left[t_{3 n}, t_{3 n+2}\right]$, one can calculate it by using a cubic interpolation. Then, we can have the following:

$$
\begin{aligned}
& F_{1,3 n+2}\left(t_{3 n+2}\right) \\
& =g_{1}\left(t_{3 n+2}\right) \\
& +\frac{3 h}{8 \Gamma(\alpha)}\left(K_{\alpha(1,1)}\left(t_{3 n+2}, t_{0}, F_{1,0}\right)\right. \\
& +3 K_{\alpha(1,1)}\left(t_{3 n+1}, t_{1}, F_{1,1}\right) \\
& +3 K_{\alpha(1,1)}\left(t_{3 n+2}, t_{2}, F_{1,2}\right)
\end{aligned}
$$




$$
\begin{array}{ll}
+2 K_{\alpha(1,1)}\left(t_{3 n+2}, t_{3}, F_{1,3}\right)+\cdots & \text { 5. Convergence Analysis of } \\
\left.+K_{\alpha(1,1)}\left(t_{3 n+2}, t_{3 n}, F_{1,3 n}\right)\right) & \text { Simpson's } 3 / 8 \text { Rule for Solving the Volterra } \\
& \text { Fractional Integral Equations }
\end{array}
$$

\section{Convergence Analysis of \\ Simpson's 3/8 Rule for Solving the Volterra Fractional Integral Equations}$$
+\frac{3 h}{8 \Gamma(\alpha)}\left(K_{\alpha(2,1)}\left(t_{3 n+2}, t_{0}, F_{2,0}\right)\right.
$$$$
+3 K_{\alpha(2,1)}\left(t_{3 n+2}, t_{1}, F_{2,1}\right)
$$$$
+3 K_{\alpha(2,1)}\left(t_{3 n+2}, t_{2}, F_{2,2}\right)
$$$$
+2 K_{\alpha(2,1)}\left(t_{3 n+2}, t_{3}, F_{2,3}\right)+\cdots
$$$$
\left.+K_{\alpha(2,1)}\left(t_{3 n+1}, t_{3 n}, F_{2,3 n}\right)\right)
$$$$
+\frac{h}{4 \Gamma(\alpha)}\left(K_{\alpha(1,1)}\left(t_{3 n+2}, t_{3 n}, F_{1,3 n}\right)+3 K_{\alpha(1,1)}\right.
$$$$
\times\left(t_{3 n+2}, t_{3 n+2 / 3}, \frac{14}{81} F_{1,3 n}+\frac{28}{27} F_{1,3 n+2}\right.
$$$$
\left.-\frac{7}{27} F_{1,3 n+2}+\frac{4}{81} F_{1,3 n+3},\right)
$$$$
+3 K_{\alpha(1,1)}
$$$$
\times\left(t_{3 n+1}, t_{3 n+2 / 3},-\frac{5}{81} F_{1,3 n}+\frac{20}{27} F_{1,3 n+1}\right.
$$$$
\left.+\frac{10}{27} F_{1,3 n+2}-\frac{4}{81} F_{1,3 n+3}\right)
$$$$
\left.+K_{\alpha(1,1)}\left(t_{3 n+2}, t_{3 n+1}, F_{1,3 n}\right)\right)
$$$$
+\frac{h}{8 \Gamma(\alpha)}\left(K_{\alpha(1,2)}\left(t_{3 n+2}, t_{3 n}, F_{2,3 n}\right)+3 K_{\alpha(1,2)}\right.
$$$$
\times\left(t_{3 n+1}, t_{3 n+2 / 3}, \frac{40}{81} F_{2,3 n}-\frac{20}{27} F_{2,3 n+2}\right.
$$$$
\left.-\frac{8}{27} F_{2,3 n+2}+\frac{5}{81} F_{2,3 n+3}\right)
$$$$
+3 K_{\alpha(1,2)}
$$$$
\times\left(t_{3 n+1}, t_{3 n+2 / 3}, \frac{14}{81} F_{2,3 n+1}\right.
$$$$
\left.-\frac{7}{27} F_{2,3 n+2}+\frac{4}{81} F_{2,3 n+3}\right)
$$$$
\left.+K_{\alpha(1,2)}\left(t_{3 n+2}, t_{3 n+2}, F_{2,3 n+3}\right)\right) \text {. }
$$

Using the similar formulas, we can obtain $F_{1,3 n+3}$ and $F_{2,3 n+3}$. Now, from (58) to (62), we formed a system of six equations with normally six unknowns for $n=1,2, \ldots$.

In particular, we do have six simultaneous equations for each step. Our next concern in this work is to show the convergence analysis of Simpson's 3/8 rule for solving the Volterra fractional integral equations, and this will be presented in the next section.
This section is devoted to the discussion underpinning the convergence of the well-known Simpson's 3/8 rule to approximate the Volterra fractional equation of second kind. There are also other numerical methods to deal with these equations [32,34-36]. To achieve this, and without loss of generality, we assume that the error in approximating the solution of the Volterra fractional equation of second kind via Simpson's $3 / 8$ rule is $R_{1,3 n+1}$ for the first approximation in (60); the rest can be obtained similarly; then,

$$
\begin{aligned}
& \left|R_{1,3 n+1}\right| \\
& =\left|F_{1,3 n+1}-f_{1}\left(x_{3 n+1}\right)\right| \\
& \leq \frac{1}{\Gamma(\alpha)} \mid h \sum_{k=0}^{3 m} \psi_{k} K_{\alpha(1,1)} \\
& \times\left(t_{3 n+1}, t_{k}, F_{1, k}\right) \\
& +h \sum_{k=0}^{3 m} \psi_{k} K_{\alpha(1,2)} \\
& \times\left(t_{3 n+1}, t_{k}, F_{2, k}\right) \\
& +\frac{h}{8 \Gamma(\alpha)} \\
& \times\left(K_{\alpha(1,1)}\left(t_{3 n+1}, t_{3 n}, F_{1,3 n}\right)+3 K_{\alpha(1,1)}\right. \\
& \times\left(t_{3 n+1}, t_{3 n+1 / 3}, \frac{40}{81} F_{1,3 n}\right. \\
& -\frac{20}{27} F_{1,3 n+1}-\frac{8}{27} F_{1,3 n+2} \\
& \left.+\frac{5}{81} F_{1,3 n+3}\right) \\
& +3 K_{\alpha(1,1)} \\
& \times\left(t_{3 n+1}, t_{3 n+2 / 3}, \frac{14}{81} F_{1,3 n+1}\right. \\
& \left.-\frac{7}{27} F_{1,3 n+2}+\frac{4}{81} F_{1,3 n+3}\right) \\
& \left.+K_{\alpha(1,1)}\left(t_{3 n+1}, t_{3 n+1}, F_{1,3 n}\right)\right) \\
& +\frac{h}{8 \Gamma(\alpha)} \\
& \times\left(K_{\alpha(1,2)}\left(t_{3 n+1}, t_{3 n}, F_{2,3 n}\right)+3 K_{\alpha(1,2)}\right.
\end{aligned}
$$




$$
\begin{aligned}
& \times\left(t_{3 n+1}, t_{3 n+1 / 3}, \frac{40}{81} F_{2,3 n}\right. \\
& \left.-\frac{20}{27} F_{2,3 n+1}-\frac{8}{27} F_{2,3 n+2}+\frac{5}{81} F_{2,3 n+3}\right) \\
& +3 K_{\alpha(1,2)} \\
& \times\left(t_{3 n+1}, t_{3 n+2 / 3}, \frac{14}{81} F_{2,3 n+1}\right. \\
& \left.-\frac{7}{27} F_{2,3 n+2}+\frac{4}{81} F_{2,3 n+3}\right) \\
& +\frac{h}{8 \Gamma(\alpha)} \\
& \times\left(K_{\alpha(1,2)}\left(t_{3 n+2}, t_{3 n}, F_{2,3 n}\right)\right. \\
& +3 K_{\alpha(1,2)} \\
& \times\left(t_{3 n+1}, t_{3 n+2 / 3}, \frac{40}{81} F_{2,3 n}\right. \\
& \left.-\frac{20}{27} F_{2,3 n+2}-\frac{8}{27} F_{2,3 n+2}+\frac{5}{81} F_{2,3 n+3}\right) \\
& +3 K_{\alpha(1,2)} \\
& \times\left(t_{3 n+1}, t_{3 n+2 / 3}, \frac{14}{81} F_{2,3 n+1}\right. \\
& \left.-\frac{7}{27} F_{2,3 n+2}+\frac{4}{81} F_{2,3 n+3}\right) \\
& \left.+K_{\alpha(1,2)}\left(t_{3 n+2}, t_{3 n+2}, F_{2,3 n+3}\right)\right) \\
& +\frac{h}{8} K_{1,2} \\
& \times\left(x_{3 n+1}, x_{3 n+1}, F_{2,3 n+1}\right) \\
& -\sum_{l=1}^{2} \int_{0}^{3 n+1} K_{1, l}\left(t_{3 n+1}, \tau, f_{l}(\tau)\right) d \tau \mid .
\end{aligned}
$$

Now, employing the Lipchitz condition for the fractional kernel function, we can arrive at the following:

$$
\begin{aligned}
\left|R_{1,3 n+1}\right| \leq & \frac{h}{\Gamma(\alpha)} a_{1} \sum_{k=0}^{3 n}\left|R_{1, k}\right|+\frac{h}{\Gamma(\alpha)} a_{2} \\
& \times \sum_{k=0}^{3 n}\left|R_{2, k}\right|+\frac{h}{\Gamma(\alpha)} a_{3}\left|R_{1,3 n+1}\right| \\
& +\frac{h}{\Gamma(\alpha)} a_{4}\left|R_{2,3 n+1}\right|+\frac{h}{\Gamma(\alpha)} a_{5}\left|R_{1,3 n+2}\right|
\end{aligned}
$$

$$
\begin{gathered}
+\frac{h}{\Gamma(\alpha)} a_{7}\left|R_{1,3 n+3}\right|+\frac{h}{\Gamma(\alpha)} a_{8}\left|R_{2,3 n+3}\right| \\
+\frac{1}{\Gamma(\alpha)}\left(\left|w_{1,3 n+1}\right|+\left|w_{2,3 n+1}\right|\right. \\
\left.+\left|w_{1,3 n+2}\right|+\left|w_{2,3 n+2}\right|\right) .
\end{gathered}
$$

Here, it is important to recall that $w_{k, 3 n+1}, w_{k, 3 n+2}(k=1,2)$ are the errors of integration rule. In addition, without loss of generality, we assume that

$$
\left\|R_{k, i}\right\|_{\infty}=\max _{k=1,2 i=3 n+1,3 n+2,3 n+3} \max _{k, i}|=| R_{1,3 n+1} \mid ;
$$

thus, by letting $A=\max _{m}\left[\left|w_{1, m}\right|,\left|w_{2, m}\right|\right]$, consequently

$$
\begin{aligned}
\left\|R_{k, i}\right\|_{\infty} \leq \frac{h}{\Gamma(\alpha)} a \sum_{n=0}^{3 n} & \left(\left|R_{1, n}\right|,\left|R_{2, n}\right|\right) \\
& +\frac{6 h}{\Gamma(\alpha)} a^{\prime}\left\|R_{k, i}\right\|_{\infty}+4 A .
\end{aligned}
$$

Now by rearranging, we obtain the following inequality:

$$
\begin{aligned}
\left\|R_{k, i}\right\|_{\infty} \leq & \frac{(h / \Gamma(\alpha)) a}{1-(6 h / \Gamma(\alpha)) a^{\prime}} \\
& \times \sum_{n=0}^{3 n}\left(\left|R_{1, n}\right|,\left|R_{2, n}\right|\right)+\frac{4 A}{1-(6 h / \Gamma(\alpha)) a^{\prime}} .
\end{aligned}
$$

However, making use of the so-called Gronwall inequality, we arrive at

$$
\left\|R_{k, i}\right\|_{\infty} \leq \frac{4 A}{1-(6 h / \Gamma(\alpha)) a^{\prime}} \operatorname{Exp}\left[\frac{h a}{1-(6 h / \Gamma(\alpha)) a^{\prime}} x_{n}\right] .
$$

For the fractional kernel function $K_{\alpha}$ and $F$ with at least fourth-order derivatives, we have $A=O\left(h^{4}\right)$ and then $\left\|R_{k, i}\right\|_{\infty}=O\left(h^{4}\right)$. Therefore, we can state the following theorem.

Theorem 8. Simpson's $3 / 8$ rule for solving the Volterra fractional integral equations of second kind is convergent and its order of convergence is at least four.

\section{Numerical Solutions}

In this section, we present some numerical examples of solutions of the Volterra fractional integral equations via the so-called Simpson's 3/8 rule.

Example 9. Let us consider the following Volterra fractional integral equation for which the order is half:

$$
f(x)=2 \sqrt{x}-\int_{0}^{x} \frac{f(t)}{\sqrt{x-t}} d x=x, \quad 0 \leq t<x<1 .
$$

The exact solution of this equation is given as

$$
f(x)=1-\exp [\pi x] \text { er } f(\sqrt{x \pi}) .
$$


TABLE 1: Numerical errors corresponding to the value of $h$.

\begin{tabular}{|c|c|c|c|c|c|}
\hline \multirow{3}{*}{$x$} & \multicolumn{5}{|c|}{$h$} \\
\hline & 0.001 & 0.01 & 0.02 & 0.04 & 0.05 \\
\hline & Error $(1) \times 10^{-5}$ & Error $(2) \times 10^{-5}$ & Error $(3) \times 10^{-5}$ & Error $(5) \times 10^{-5}$ & Error $(6) \times 10^{-5}$ \\
\hline 0 & 0.01 & 0.02 & 0.03 & 0.04 & 0.05 \\
\hline 0.1 & 0.00216833 & 0.00433666 & 0.00650499 & 0.00867332 & 0.0108417 \\
\hline 0.2 & 0.003822808 & 0.00765615 & 0.0114842 & 0.0153123 & 0.0191404 \\
\hline 0.3 & 0.0113064 & 0.0226128 & 0.339192 & 0.0452256 & 0.0565321 \\
\hline 0.4 & 0.0211693 & 0.0423387 & 0.063508 & 0.0846773 & 0.105847 \\
\hline 0.5 & 0.0344335 & 0.0688669 & 0.1033 & 0.137734 & 0.172167 \\
\hline 0.6 & 0.0524239 & 0.104848 & 0.157272 & 0.209695 & 0.262119 \\
\hline 0.7 & 0.0769263 & 0.157272 & 0.230779 & 0.307705 & 0.384631 \\
\hline 0.8 & 0.110371 & 0.220743 & 0.331114 & 0.441485 & 0.551856 \\
\hline 0.9 & 0.156078 & 0.312156 & 0.468234 & 0.624312 & 0.780391 \\
\hline 1 & 0.218586 & 0.437173 & 0.655759 & 0.874345 & 1.09293 \\
\hline
\end{tabular}

Exact-approx-for $h=0.001$

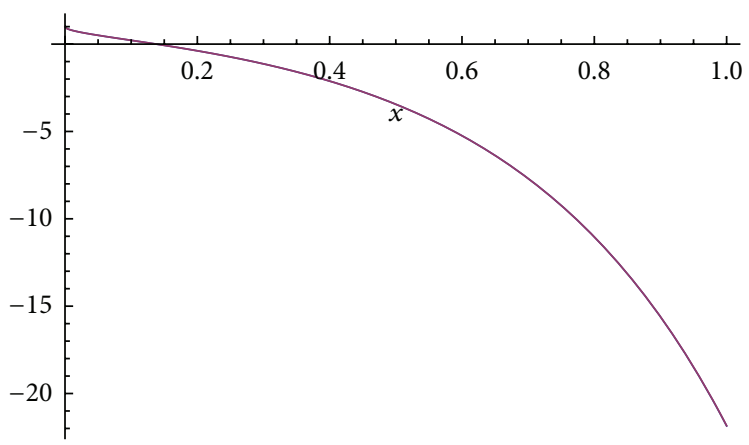

FIGURE 1: Comparison of exact solution and approximate solution for $h=0.001$.

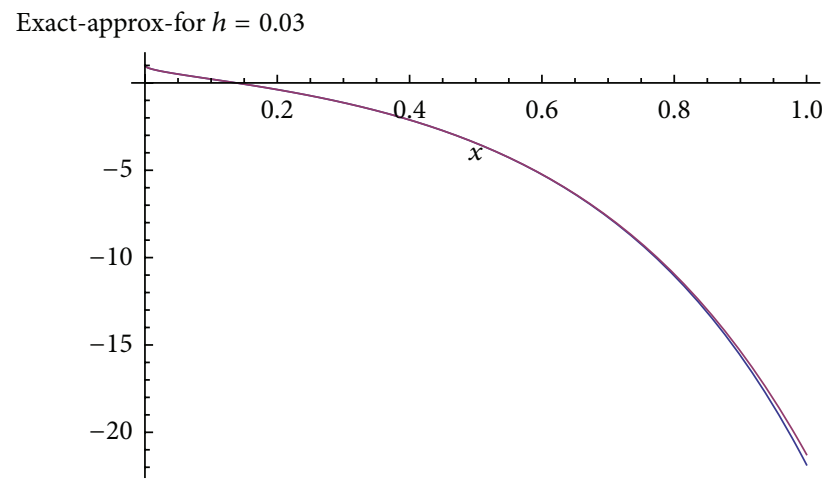

FIGURE 2: Comparison of exact solution and the approximate solution for $h=0.03$.

Using the Simpson 3/8, we obtained the following numerical values indicated in Table 1 .

The approximate solutions have been depicted in Figures 1,2 , and 3 . Figure 1 shows the comparison of the exact and approximate solutions for $h=0.001$, Figure 2 shows the

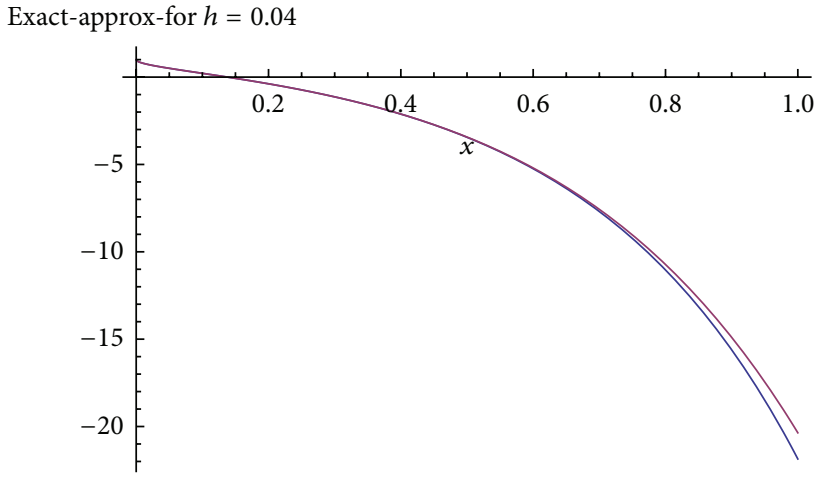

FIGURE 3: Comparison of the exact and approximate solution for $h=$ 0.04 .

comparison for $h=0.03$, and Figure 3 shows the comparison for $h=0.04$. The numerical solution shows that the method is very efficient and accurate.

\section{Conclusion}

The existence and the uniqueness of the Volterra fractional integral equations second kind were examined in this work. The numerical method called the Simpson 3/8 rule method was used to present the numerical solution of these equations. We presented the convergence analysis of this numerical scheme.

\section{Conflict of Interests}

The authors declare no conflict of interests.

\section{Author's Contribution}

Abdon Atangana wrote the first draft and Necdet Bildik corrected the revised form; the both authors read the revised and submitted the paper. 


\section{References}

[1] K. E. Atkinson, The Numerical Solution of Integral Equations of the Second Kind, Cambridge University Press, 1997.

[2] E. Babolian and J. Biazar, "Solution of a system of non-linear Volterra integral equations of the second kind," Far East Journal of Mathematical Sciences, vol. 2, no. 6, pp. 935-945, 2000.

[3] P. Linz, Analytical and Numerical Methods for Volterra Equations, SIAM, Philadelphia, Pa, USA, 1985.

[4] K. Maleknejad and M. Karami, "Numerical solution of nonlinear Fredholm integral equations by using multiwavelets in the Petrov-Galerkin method," Applied Mathematics and Computation, vol. 168, no. 1, pp. 102-110, 2005.

[5] A. Atangana and A. Kilicman, "Analytical solutions of the spacetime fractional derivative of advection dispersion equation," Mathematical Problems in Engineering, vol. 2013, Article ID 853127, 9 pages, 2013.

[6] M. M. Meerschaert and C. Tadjeran, "Finite difference approximations for fractional advection-dispersion flow equations," Journal of Computational and Applied Mathematics, vol. 172, no. 1, pp. 65-77, 2004.

[7] M. Caputo, "Linear models of dissipation whose Q is almost frequency independent-part II," Geophysical Journal International, vol. 13, no. 5, pp. 529-539, 1967.

[8] A. Cloot and J. F. Botha, "A generalised groundwater flow equation using the concept of non-integer order derivatives," Water SA, vol. 32, no. 1, pp. 55-78, 2006.

[9] D. A. Benson, S. W. Wheatcraft, and M. M. Meerschaert, "Application of a fractional advection-dispersion equation," Water Resources Research, vol. 36, no. 6, pp. 1403-1412, 2000.

[10] A. Atangana and A. Kılıçman, "A possible generalization of acoustic wave equation using the concept of perturbed derivative order," Mathematical Problems in Engineering, vol. 2013, Article ID 696597, 6 pages, 2013.

[11] F. Mainardi, "Fractional calculus: some basic problems in continuum and statistical mechanics," in Fractals and Fractional Calculus in Continuum Mechanics, vol. 378 of CISM Courses and Lectures, pp. 291-348, Springer, Vienna, Austria, 1997.

[12] P. Zhuang, F. Liu, V. Anh, and I. Turner, "Numerical methods for the variable-order fractional advection-diffusion equation with a nonlinear source term," SIAM Journal on Numerical Analysis, vol. 47, pp. 1760-1781, 2009.

[13] S. B. Yuste and L. Acedo, "An explicit finite difference method and a new von Neumann-type stability analysis for fractional diffusion equations," SIAM Journal on Numerical Analysis, vol. 42, no. 5, pp. 1862-1874, 2005.

[14] C.-M. Chen, F. Liu, I. Turner, and V. Anh, "A Fourier method for the fractional diffusion equation describing sub-diffusion," Journal of Computational Physics, vol. 227, no. 2, pp. 886-897, 2007.

[15] A. Atangana and E. Alabaraoye, "Solving a system of fractional partial differential equations arising in the model of HIV infection of CD4+ cells and attractor one-dimensional KellerSegel equations," Advances in Difference Equations, vol. 2013, article 94, 2013.

[16] A. Atangana, O. A. Ahmed, and N. Bıldık, "A generalized version of a low velocity impact between a rigid sphere and a transversely isotropic strain-hardening plate supported by a rigid substrate using the concept of non-integer derivatives," Abstract and Applied Analysis, vol. 2013, Article ID 671321, 9 pages, 2013.
[17] A. Atangana and A. Secer, "The time-fractional coupledKorteweg-de-Vries equations," Abstract and Applied Analysis, vol. 2013, Article ID 947986, 8 pages, 2013.

[18] G. C. Wu, "New trends in the variational iteration method," Communications in Fractional Calculus, vol. 2, no. 2, pp. 59-75, 2011.

[19] G. C. Wu and D. Baleanu, "Variational iteration method for fractional calculus-a universal approach by Laplace transforms," Advances in Difference Equations, vol. 2013, article 18, 2013.

[20] G. C. Wu and D. Baleanu, "Variational iteration method for the Burgers' flow with fractional derivatives-New Lagrange mutipliers," Applied Mathematical Modelling, vol. 37, pp. 61836190, 2012.

[21] S. Duan, R. Rach, D. Baleanu, and A. M. Wazwaz, "A review of the Adomian decomposition method and its applications to fractional differential equations," Communications in Fractional Calculus, vol. 3, no. 2, pp. 73-99, 2012.

[22] V. Daftardar-Gejji and H. Jafari, "Adomian decomposition: a tool for solving a system of fractional differential equations," Journal of Mathematical Analysis and Applications, vol. 301, no. 2, pp. 508-518, 2005.

[23] M. Matinfar and M. Ghanbari, "The application of the modified variational iteration method on the generalized Fisher's equation," Journal of Applied Mathematics and Computing, vol. 31, no. 1-2, pp. 165-175, 2009.

[24] Y. Tan and S. Abbasbandy, "Homotopy analysis method for quadratic Riccati differential equation," Communications in Nonlinear Science and Numerical Simulation, vol. 13, no. 3, pp. 539-546, 2008.

[25] A. Anatoly, J. Juan, and M. S. Hari, Theory and Application of Fractional Differential Equations, Elsevier, Amsterdam, The Netherlands, 2006.

[26] D. Baleanu, K. Diethelm, E. Scalas, and J. J. Trujillo, Fractional Calculus Models and Numerical Methods, Complexity, Nonlinearity and Chaos, World Scientific, 2012.

[27] A. A. Kilbas, H. M. Srivastava, and J. J. Trujillo, Theory and Applications of Fractional Differential Equations,, vol. 204 of North-Holland Mathematics Studies, Elsevier Science B. V., Amsterdam, The Netherlands, 2006.

[28] K. S. Miller and B. Ross, An Introduction to the Fractional Calculus and Fractional Differential Equations, A Wiley-Interscience Publication, John Wiley \& Sons, New York, NY, USA, 1993.

[29] A. Atangana and A. Secer, "A note on fractional order derivatives and table of fractional derivatives of some special functions," Abstract and Applied Analysis, vol. 2013, Article ID 279681, 8 pages, 2013.

[30] F. B. Hildebrand, Introduction to Numerical Analysis, McGrawHill, New York, NY, USA, 1956.

[31] J. Biazar, Solving system of integral equations by Adomian decomposition method [Ph.D. thesis], Teacher Training University, 2002.

[32] R. K. Saeed and C. Ahmed, "Approximate solution for the system of non-linear Volterra integral equations of the second kind by using block-by-block method," Australian Journal of Basic and Applied Sciences, vol. 2, no. 1, pp. 114-124, 2008.

[33] M. Pate, “The naval artificer's manual: (The naval artificer's handbook revised) text, questions and general information for deck," United States Bureau of Reconstruction and Repair, 1918.

[34] M. Rabbani, K. Maleknejad, and N. Aghazadeh, "Numerical computational solution of the Volterra integral equations system of the second kind by using an expansion method," Applied 
Mathematics and Computation, vol. 187, no. 2, pp. 1143-1146, 2007.

[35] M. E. A. El Tom, "Application of spline functions to systems of volterra integral equations of the first and second kinds," IMA Journal of Applied Mathematics, vol. 17, no. 3, pp. 295-310, 1976.

[36] A. Akyüz-Daşcıloğlu, "Chebyshev polynomial solutions of systems of linear integral equations," Applied Mathematics and Computation, vol. 151, no. 1, pp. 221-232, 2004. 


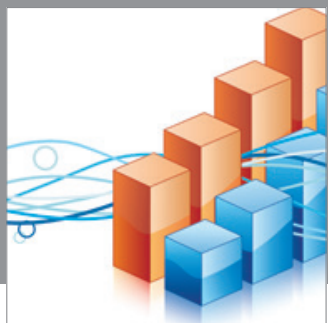

Advances in

Operations Research

mansans

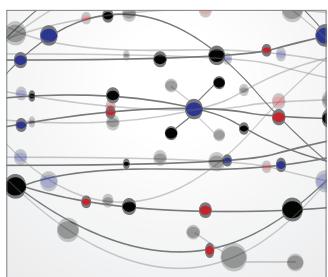

The Scientific World Journal
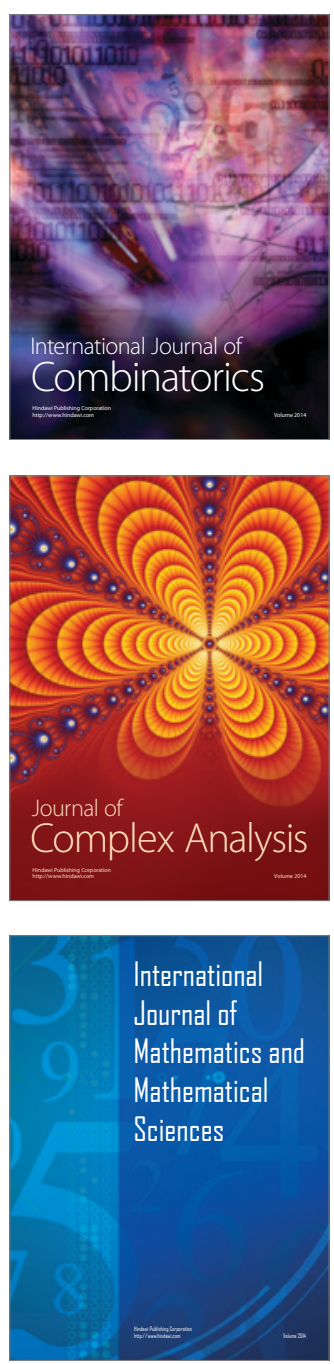
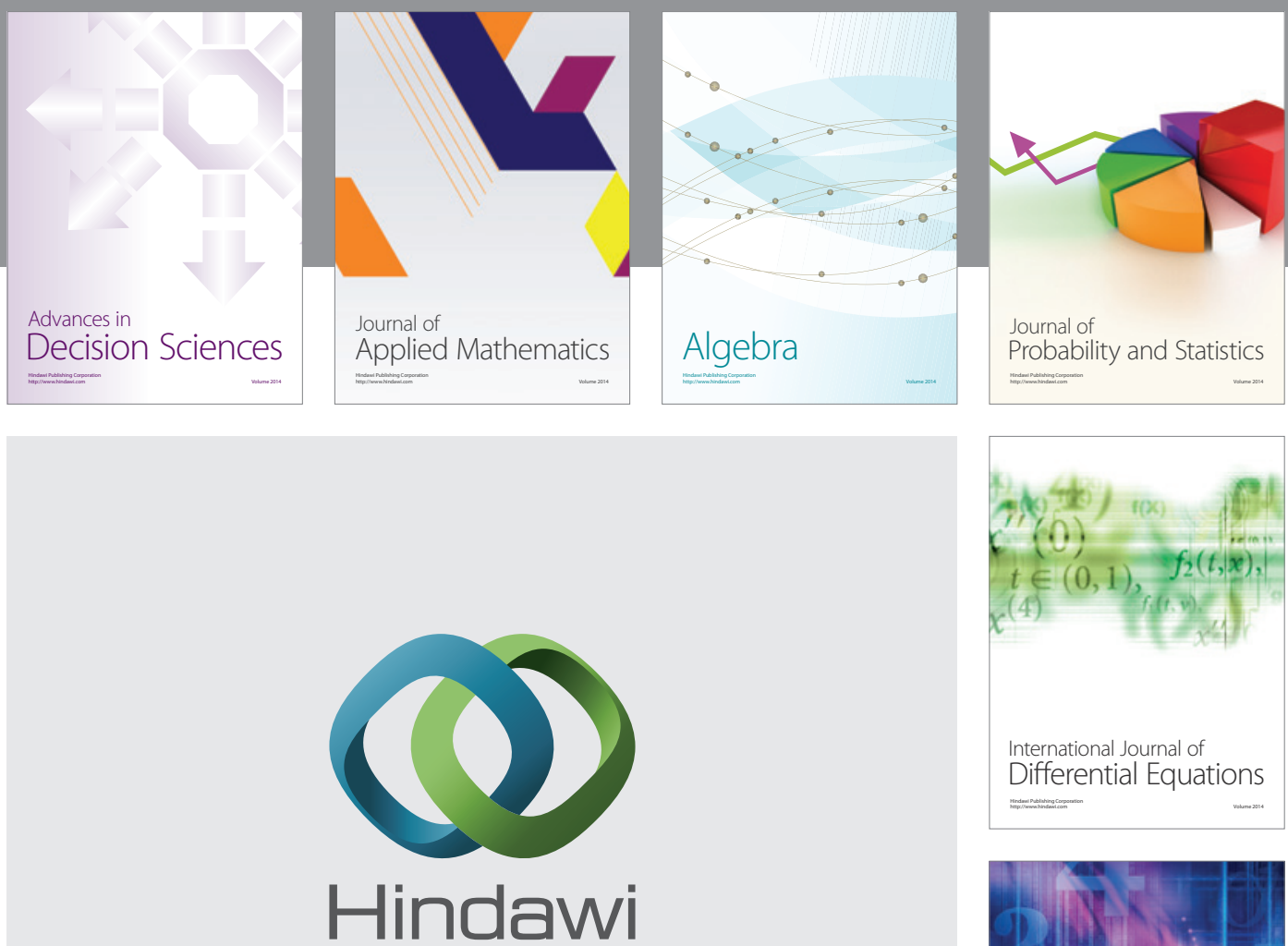

Submit your manuscripts at http://www.hindawi.com
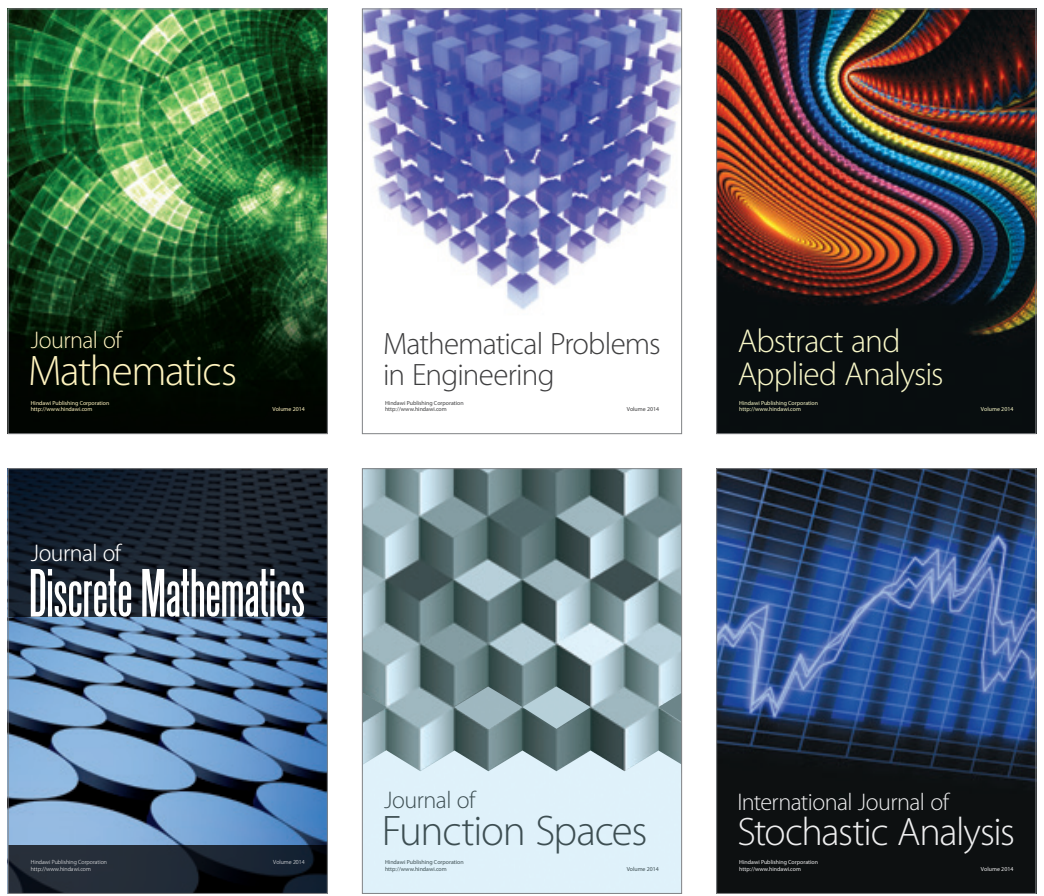

Journal of

Function Spaces

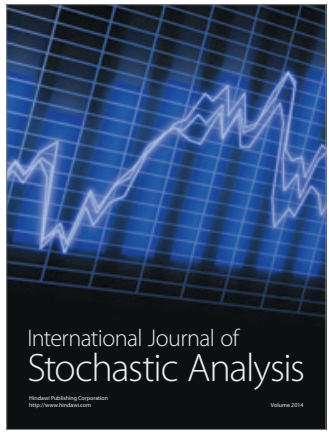

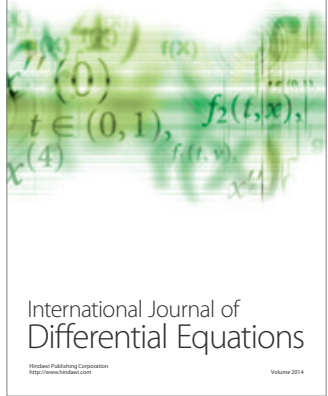
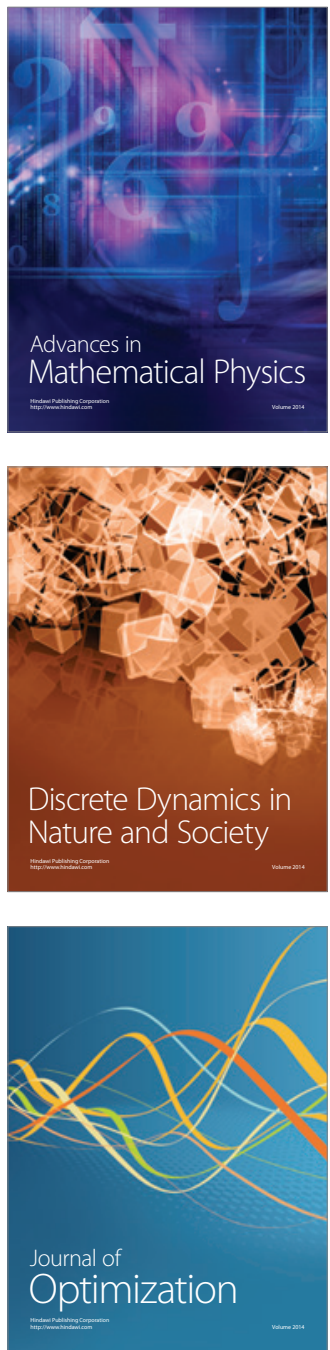